\title{
Review of Chinese Oligaphorurini (Collembola, Onychiuridae) with descriptions of two new Palaearctic species
}

\author{
Xin Sun ${ }^{1, \dagger}$, Donghui Wu ${ }^{1,2, \ddagger}$ \\ I Key laboratory of Wetland Ecology and Environment, Northeast Institute of Geography and Agroecology, Chi- \\ nese Academy of Sciences, Changchun 130012, China 2 College of Earth Science, Jilin University, Changchun \\ 130061, China \\ † urn:lsid:zoobank.org:author:2E68A57F-2AE1-45F3-98B2-EEF252590DC3 \\ † urn:lsid:zoobank.org:author:3B02EBFF-8329-4872-B77F-85F89E9230D9 \\ Corresponding author: Donghui Wu (wudonghui@neigae.ac.cn) \\ Academic editor: L. Deharveng | Received 16 February 2012 | Accepted 17 April 2012 | Published 8 May 2012 \\ urn:lsid:zoobank.org:pub:30D72D39-3423-472A-8C69-B38E21008F74 \\ Citation: Sun X, Wu D (2012) Review of Chinese Oligaphorurini (Collembola, Onychiuridae) with descriptions of two \\ new Palaearctic species. ZooKeys 192: 15-26. doi: 10.3897/zookeys.192.2959
}

\begin{abstract}
A checklist of Chinese Oligaphorurini is given. Two new Chinese species, Micraphorura changbaiensis sp. n. and Oligaphorura pseudomontana sp. n., are described from Changbai Mountain Range. M. changbaiensis sp. $\mathrm{n}$. has the same dorsal pseudocelli formula and number of papillae in Ant. III sensory organ as M. uralica, but they can be easily distinguished by number of chaetae in Ant. III sensory organ, ventral pseudocelli formula, ventral parapseudocelli formula, number of pseudocelli on subcoxa 1 of legs I-III, dorsal axial chaeta on Abd. $\mathrm{V}$ and number of chaetae on tibiotarsi. O. pseudomontana sp. n. is very similar to the species $O$. montana having an increased number of pseudocelli on body dorsally, well marked base of antenna with 1 pseudocellus and 3 pseudocelli outside, subcoxa 1 of legs I-III with 1 pseudocellus each, dorsally S-chaetae formula as 11/011/22211 from head to Abd. V, S-microchaeta present on Th. II-III, claw without inner teeth and with $1+1$ lateral teeth, and unguiculus with basal lamella; but they can be separated easily by the number of pseudocelli on Abd. V and VI terga, parapseudocelli on the body, number of chaetae on Th. I tergum, and number of chaetae on tibiotarsi. A key to Chinese species of Oligaphorurini is provided in the present paper.
\end{abstract}

\section{Keywords}

Collembola, identification key, Micraphorura changbaiensis sp. n., Oligaphorura pseudomontana sp. n., taxonomy

Copyright Xin Sun, Donghui Wu. This is an open access article distributed under the terms of the Creative Commons Attribution License 3.0 (CC-BY), which permits unrestricted use, distribution, and reproduction in any medium, provided the original author and source are credited. 


\section{Introduction}

The tribe Oligaphorurini, erected by Bagnall (1949) as a subfamily, is characterized by having a small postantennal organ with a 3-5 lobed vesicle. So far, 38 species belonging to five genera were reported in the world (Bellinger et al. 2012). Nevertheless, the Chinese fauna of Oligaphorurini is poorly known, only two species, Dimorphaphorura sanjiangensis Sun \& Wu, 2012 and Oligaphorura ursi (Fjellberg, 1984), were reported from northeast China (Sun and Wu 2012).

In the present paper, two new Chinese Oligaphorurini species are described from Changbai Mountain Range in Jilin Province, and two newly recorded species, Oligaphorura judithae (Weiner, 1994) and Oligaphorura koreana (Weiner, 1994), are mentioned. A checklist of Chinese Oligaphorurini and an identification key to all Chinese species of this tribe are given below.

\section{Material and methods}

Specimens were mounted in Marc André II solution, after clearing in lactic acid, and were studied using a Nikon Eclipse 80i microscope. Material is deposited in the Key laboratory of Wetland Ecology and Environment, Northeast Institute of Geography and Agroecology, Chinese Academy of Sciences, Changchun.

\section{Abbreviations used in descriptions}

Labial papillae types are named after Fjellberg (1999). Labium areas and chaetal nomenclature follow Massoud (1967) and D'Haese (2003). Chaetae on anal valves are named after Yoshii (1996).

Ant.-antennal segments, PAO-postantennal organ, Th.-thoracic segments, Abd.abdominal segments, p-chaeta-chaeta of row p, Sp-posterior S-chaeta (e.g. on Abd. $\mathrm{V}$ or on head), ms-S-microchaeta, pso-pseudocellus, a-pso-postero-internal pso on head, psx-parapseudocellus, psp-pseudopore, $\mathrm{x}$-axial pseudopore of Abd. IV.

Pseudocelli, parapseudocelli and pseudopore formula are the number of pseudocelli, parapseudocelli or pseudopores by half tergum (dorsally) or half-sternum (ventrally) as follows: head anterior, head posterior/Th. I, Th. II, Th. III/Abd. I, Abd. II, Abd. III, Abd. IV, Abd. V (for instance: 43/144/54464).

$\mathrm{S}$-chaetae formula is the number of S-chaetae by half tergum from head to Abd. VI (for instance: 11/011/222111).

Tibiotarsus chaetotaxy formula: total number of chaetae (number of basal chaetae, number of chaetae in row $B$, number of chaetae in distal row $A+T$, for instance: 19 $(1,7,11)$. 
Checklist of Chinese species of Oligaphorurini Bagnall, 1949

Dimorphaphorura sanjiangensis Sun \& Wu, 2012

Distribution. Heilongjiang Province (according to the original paper), Jilin Province (Changbai Mountain Range, alt. $689 \mathrm{~m}, 43.037640^{\circ} \mathrm{N}, 128.199653^{\circ} \mathrm{E}$ ).

Micraphorura changbaiensis sp. $\mathbf{n}$.

Distribution. Jilin Province.

Oligaphorura judithae (Weiner, 1994) newly recorded in China

Distribution. Jilin Province (Changbai Mountain Range, alt. $689 \mathrm{~m}, 43.037640^{\circ} \mathrm{N}$, $\left.128.199653^{\circ} \mathrm{E}\right)$; North Korea (according to the original paper).

Oligaphorura koreana (Weiner, 1994) newly recorded in China

Distribution. Jilin Province (Changbai Mountain Range, alt. $689 \mathrm{~m}, 43.037640^{\circ} \mathrm{N}$, $128.199653^{\circ} \mathrm{E}$ and alt. $1763 \mathrm{~m}, 41.755265^{\circ} \mathrm{N}, 127.941123^{\circ} \mathrm{E}$ ); North Korea (according to the original paper).

\section{Oligaphorura ursi (Fjellberg, 1984)}

Distribution. Jilin Province (Changbai Mountain Range, alt. $689 \mathrm{~m}, 43.037640^{\circ} \mathrm{N}$, $128.199653^{\circ} \mathrm{E}$ ), Heilongjiang Province (according to Sun and Wu 2012); Northern Holarctic (according to the original paper).

\section{Oligaphorura pseudomontana sp. n.}

Distribution. Jilin Province.

Key to Chinese species of Oligaphorurini Bagnall, 1949

1 Furca reduced to a finely granulated area, with $1+1$ dental chaetae posteriorly (Dimorphaphorura Bagnall, 1949).

Dimorphaphorura sanjiangensis Sun \& Wu, 2012

- $\quad$ Furca reduced to a small cuticular fold, with $1+1$ or $2+2$ dental chaetae posteriorly

$21+1$ dental chaetae posteriorly (Micraphorura Bagnall, 1949) Micraphorura changbaiensis sp. $\mathbf{n}$.

$2+2$ dental chaetae in two rows posteriorly (Oligaphorura Bagnall, 1949) ...3 First thoracic tergum with $0+0$ pso

Oligaphorura koreana (Weiner, 1994)

- $\quad$ First thoracic tergum with $1+1$ pso 4

4 The base of antenna with $4+4$ pso ...... Oligaphorura pseudomontana sp. n. 
Lateral ms absent on Th. III, $5+5$ pso on Abd. IV and $4+4$ pso on Abd. V ... Oligaphorura judithae (Weiner, 1994) - $\quad$ Lateral ms present on Th. III, 4+4 pso on Abd. IV and 3+3 pso on Abd. V .. Oligaphorura ursi (Fjellberg, 1984)

\section{Systematics}

\section{Micraphorura changbaiensis sp. $\mathrm{n}$.} urn:lsid:zoobank.org:act:E9432AE4-B11B-4F86-B581-BC15BC6A85E8 http://species-id.net/wiki/Micraphorura_changbaiensis

Figs 1-2

Type material. Holotype female, 4 female and 1 male paratypes. China: Jilin Province: Changbai Mountain Range (alt. $\left.1763 \mathrm{~m}, 41.755265^{\circ} \mathrm{N}, 127.941123^{\circ} \mathrm{E}\right): 15 . V I I I .2009$, litter and soil, Berlese extraction, Wu Dong-hui leg.

Holotype and 5 paratypes on slides are deposited in the Key laboratory of Wetland Ecology and Environment, Northeast Institute of Geography and Agroecology, Chinese Academy of Sciences, Changchun.

Diagnosis. Pso formula as 32/133/44454 dorsally and 11/000/00000 ventrally; subcoxa 1 of legs I-III with 1 pso each; psx formula as 00/000/222200 ventrally, absent dorsally; S-chaetae formula as 11/011/222111 dorsally and 11/000/000100 ventrally; Ant. III sensory organ composed of 5 papillae, 5 guard chaetae, 2 small sensory rods, 2 granulated sensory clubs; Abd. V tergum with one dorsal axial chaeta (p0), Abd. VI with two axial chaetae (a0 and p0); tibiotarsi of legs I, II and III with 20, 20 and 19 chaetae; anal spines present on indistinct papillae, as long as inner edge of unguis.

Description. Body color white in alcohol. Size $0.70-0.82 \mathrm{~mm}$, holotype: 0.78 $\mathrm{mm}$. Body slender and elongated.

Pseudocelli (pso) formula as 32/133/44454 dorsally and 11/000/00000 ventrally (Figs 1A, 2C); subcoxa 1 of legs I-III with 1 pso each. Parapseudocelli (psx) formula as 00/000/222200 ventrally and absent dorsally (Figs 1A, 2C); subcoxa 1 of legs I-III with 2 psx each. Pseudopore (psp) formula as 00/011/111100 dorsallyand 00/111/000x00 ventrally (Figs 1A, 2C).

S-chaetae cylindrical, well differentiated, formula as 11/011/222111 dorsallyand 11/000/000100 ventrally (Figs 1A, 2C); subcoxae 2 of legs I, II and III with 0,0 and 1 S-chaeta respectively (Fig. 2D). Two posterior S-chaetae (Sp) present on head. Smicrochaetae tiny and blunt, present on Th. II-III (Fig. 1A).

Head. Antennae short and distinctly segmented, 0.8 times as long as head. Length ratio of antennal segments I: II: III: IV = 1: 1.8: 1.8: 2. Ant. IV with two distinct thickened S-chaetae, subapical organite with apex globular and basolateral ms just above posterior chaetae (Fig. 1C). Ant. III sensory organ composed of 5 papillae, 5 guard chaetae, 2 small sensory rods, 2 granulated sensory clubs, the outer about twice as large as the inner, and a lateral ms (Fig. 1D). Ant. II with 14 chaetae. Ant. I with 8 chaetae. Antennal base without 


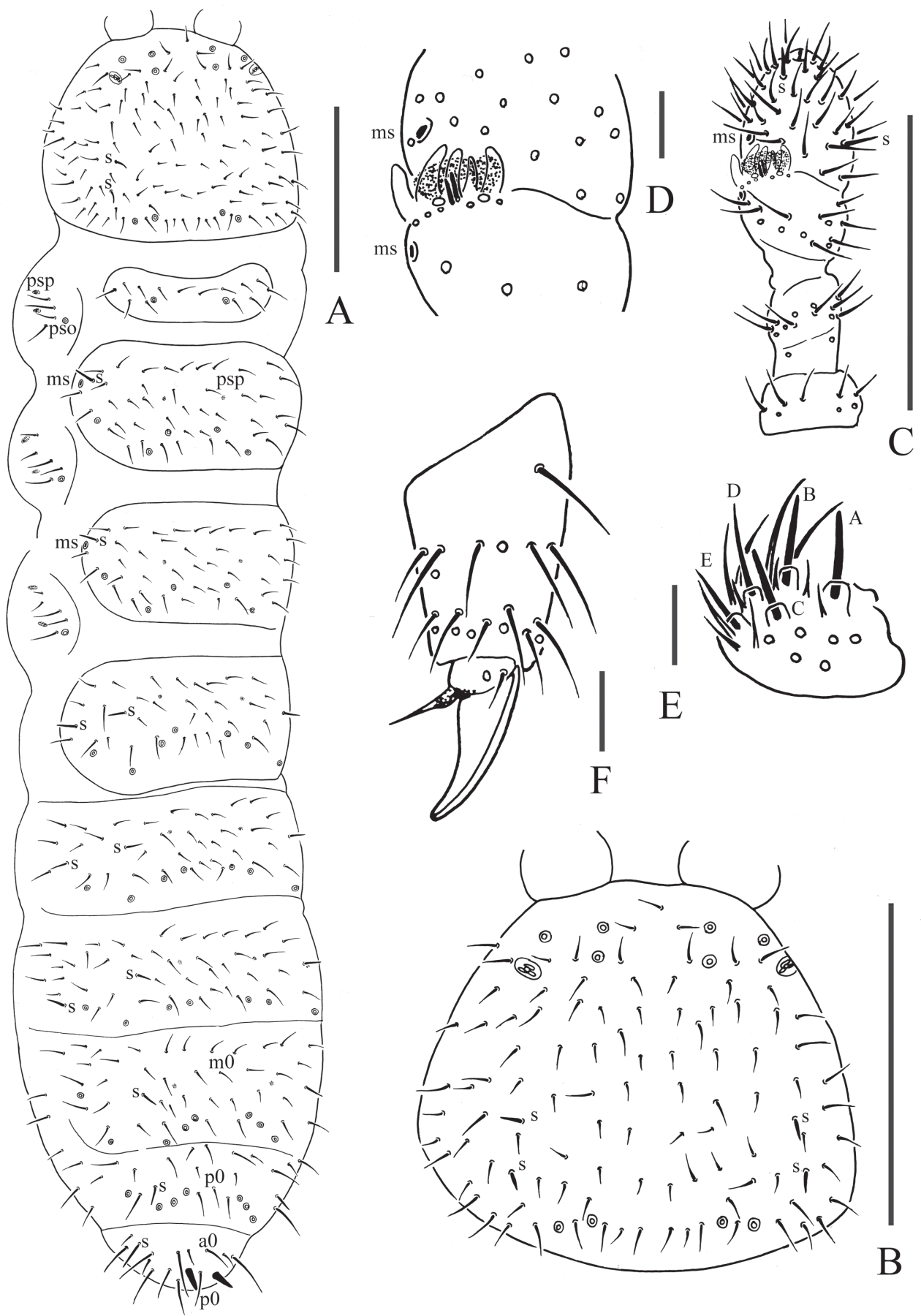

Figure I. Micraphorura changbaiensis sp. n. A dorsal side of body B dorsal side of head $\mathbf{C}$ antenna D organ of Ant. III E labium $\mathbf{F}$ distal part of leg III. Scales: $0.1 \mathrm{~mm}(\mathrm{~A}-\mathrm{C}), 0.01$ (D-F) 

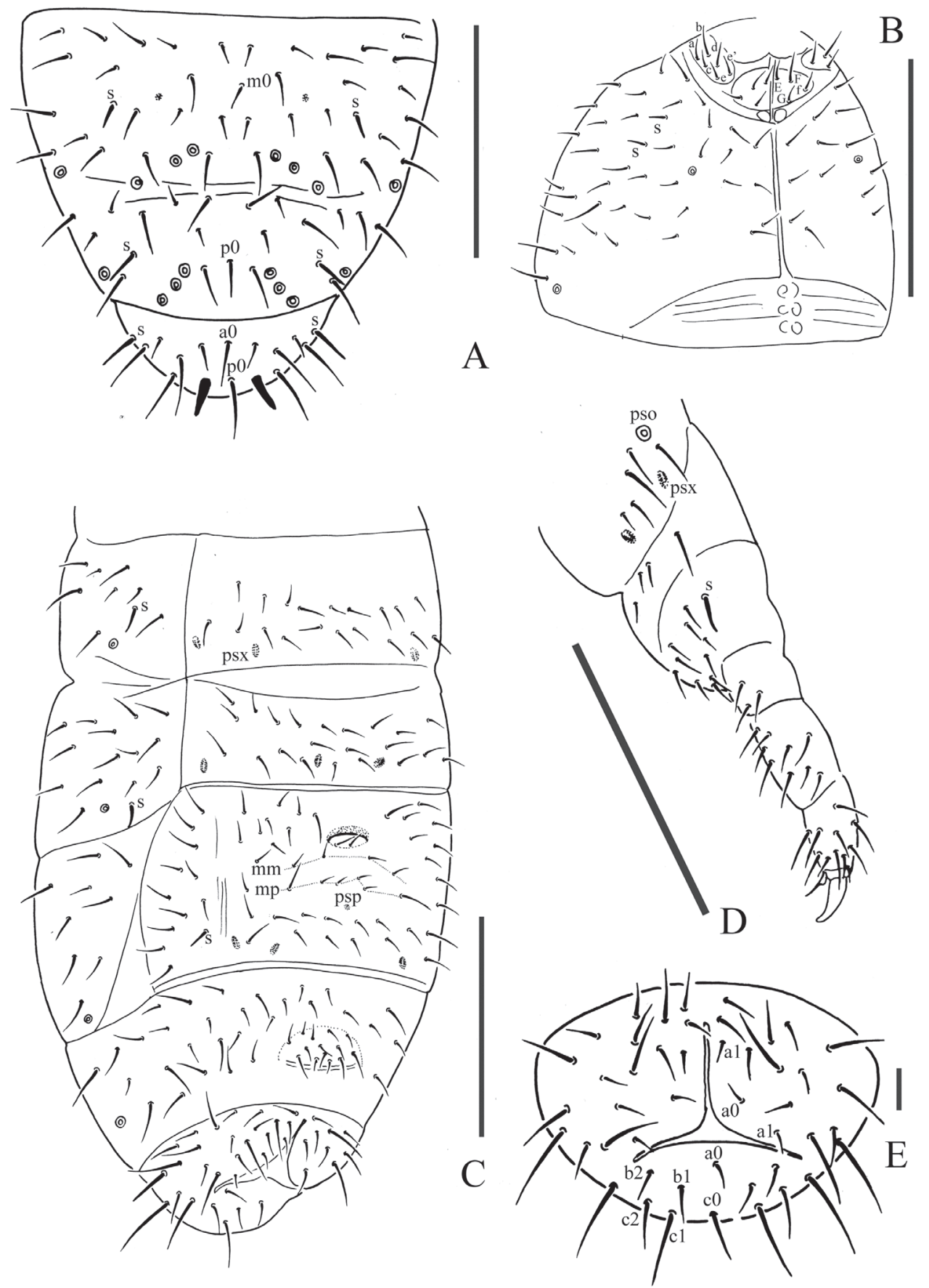

Figure 2. Micraphorura changbaiensis sp. n. A dorsal side of Abd. IV-VI B ventral side of head $\mathbf{C}$ ventral side of Abd. II-VI D leg III E anal valves. Scales: $0.1 \mathrm{~mm}$ (A-D), 0.01 (E) 
distinct granulation. PAO located on cuticular furrow built with a 3-4 lobed vesicle (Fig. 1B). Dorsal cephalic chaeta d0 absent, $3+3$ p-chaetae between posterior a-pso on head (Fig. 1B). Mandible with strong molar plate and 4 apical teeth. Maxilla bearing 3 teeth and 6 lamellae. Maxillary palp simple with 1 basal chaeta and 2 sublobal hairs. Labral chaetae formula 4/342. Labium with 6 proximal, 4 basomedian (E, F, G, f) and 6 basolateral (a, b, c, d, e, e') chaetae (Fig. 2B); labial type ABC, papillae A-E respectively with 1, 4, 0, 3, 3 guard chaetae (Fig. 1E). Postlabial chaetae 4+4 along ventral groove (Fig. 2B).

Body chaetotaxy. Ordinary chaetae differentiated in meso- and macrochaetae. Th. I tergum with 7+7 chaetae dorsally (Fig. 1A). Th. II-III and Abd. I-III terga with three chaetae on both side of axial line and no dorsal axial chaetae. Abd. IV tergum with one dorsal axial chaeta $(\mathrm{m} 0)$, Abd. V with one dorsal axial chaeta $(\mathrm{p} 0)$, Abd. VI with two axial chaetae (a0 and p0) (Fig. 2A). Th. I, II and III sterna with $0+0 / 1+1 / 1+1(2+2)$ chaetae.

Appendages. Subcoxa 1 of legs I-III with 4, 5 and 5 chaetae, subcoxa 2 with 1 , 4 and 4 chaetae respectively. Tibiotarsi of legs I, II and III with $20(1,8,11), 20$ (1, $8,11)$ and $19(1,7,11)$ chaetae (Fig. 1F). Unguis without teeth. Unguiculus slender and pointed, 0.6 times as long as inner edge of unguis, with inner basal lamella (Figs $1 F, 2 D)$. Ventral tube with $6+6$ distal chaetae and $2+2$ basal chaetae, without anterior chaetae. Furca reduced to a small cuticular fold with $1+1$ dental chaetae posteriorly; two manubrial rows of chaetae present (Fig. 2C).

Female genital plate with 14 chaetae; in our specimens, the only male is juvenile. Anal valves with numerous acuminate chaetae; each lateral valve with a0 and 2a1; upper valves with chaetae a0, 2b1, 2b2, c0, 2c1, 2c2 (Fig. 2E). Anal spines present on indistinct papillae, as long as inner edge of unguis (Fig. 1A).

Etymology. Named after the mountain range where we found the new species.

Ecology. Found in coniferous forest.

Remarks. The new species has the same dorsal pseudocelli formula (32/133/44454) and number of papillae in Ant. III sensory organ (5) as M. uralica (Khanislamova, 1986), but they can be easily distinguished by number of guard chaetae on Ant. III sensory organ ( 5 in changbaiensis sp. $\mathrm{n}$., 4 in uralica), ventral pseudocelli formulae (11/000/00000 in changbaiensis sp. n., 11/000/11120 in uralica), ventral parapseudocelli formulae (00/000/222200 in changbaiensis sp. n., indistinct in uralica), pseudocelli on subcoxa 1 of legs I-III (1, 1, 1 in changbaiensis sp. n., 2, 3, 3 in uralica), dorsal axial chaeta on Abd. V ( $\mathrm{m} 0$ in changbaiensis sp. $\mathrm{n}$., absent in uralica), and number of chaetae on tibiotarsi $(20,20,19$ in changbaiensis sp. n., 19, 19, 18 in uralica).

\section{Oligaphorura pseudomontana sp. $\mathbf{n}$.}

urn:Isid:zoobank.org:act:6691C23D-A275-4C91-B001-C4C5AFCC8D04 http://species-id.net/wiki/Oligaphorura_pseudomontana

Figs 3-4

Type material. Holotype male, 2 female and 5 male paratypes. China: Jilin Province: Changbai Mountain Range (alt. 689m, $43.037640^{\circ} \mathrm{N}, 128.199653^{\circ} \mathrm{E}$ ): 3.X.2011, litter and soil, Berlese extraction, Tang Xu-guang leg. 
Holotype and 7 paratypes on slides are deposited in the Key laboratory of Wetland Ecology and Environment, Northeast Institute of Geography and Agroecology, Chinese Academy of Sciences, Changchun.

Diagnosis. Pso formula as 43/144/54464 dorsally and 11/000/00000 ventrally; subcoxa 1 of legs I-III with 1 pso each; psx formula as 00/000/222401 ventrally and absent dorsally; S-chaetae formula as 11/011/222111 dorsally and 11/000/000100 ventrally; Ant. III sensory organ composed of 5 papillae, 5 guard chaetae, 2 small sensory rods, 2 granulated sensory clubs; Abd. V without dorsal axial chaetae, Abd. VI with two axial chaetae ( $\mathrm{a} 0$ and p0); tibiotarsi of legs I, II and III with 20, 20 and 19 chaetae; anal spines present on indistinct papillae, 0.75 times as long as inner edge of unguis.

Description. Body color white in alcohol. Size $0.80-1.04 \mathrm{~mm}$; holotype: 0.90 $\mathrm{mm}$. Body slender and elongated.

Pseudocelli (pso) formula as 43/144/54464 dorsally and 11/000/00000 ventrally (Figs 3A, E); subcoxa 1 of legs I-III with 1 pso each (Fig. 4C). Parapseudocelli (psx) formula as 00/000/222401 ventrally and absent dorsally (Figs 3A, E); subcoxa 1 of legs I-III with 1 psx each (Fig. 4C). Pseudopore (psp) formula as 00/011/111100 dorsally and $00 / 111 / 000 x 00$ ventrally (Figs $3 A, E$ ).

S-chaetae cylindrical, well differentiated, formula as 11/011/222111 dorsally and 11/000/000100 ventrally (Figs 3A, E); subcoxae 2 of legs I, II and III with 0, 0 and 1 S-chaeta respectively (Fig. 4C). Two posterior S-chaetae (Sp) present on head. Smicrochaetae tiny and blunt, present on Th. II-III (Fig. 3A).

Head. Antennae short and distinctly segmented, as long as head. Length ratio of antennal segments I: II: III: IV = 1:2:2: 2 . Ant. IV with two distinct thickened S-chaetae, subapical organite with apex globular and basolateral ms just above posterior chaetae (Fig. 3C). Ant. III sensory organ composed of 5 papillae, 5 guard chaetae, 2 small sensory rods, 2 granulated sensory clubs, the outer about twice as large as the inner, and a lateral ms (Fig. 3B). Ant. II with 15 chaetae. Ant. I with 9 chaetae. Antennal base with distinct granulation. PAO located on cuticular furrow built with a 3 lobed vesicle (Fig. 4A). Dorsal cephalic chaeta d0 absent, $3+3$ p-chaetae between posterior a-pso on head (Fig. 4A). Mandible with strong molar plate and 4 apical teeth. Maxilla bearing 3 teeth and 6 lamellae. Maxillary palp simple with 1 basal chaeta and 2 sublobal hairs. Labral chaetae formula 4/342. Labium with 6 proximal, 4 basomedian (E, F, G, f) and 6 basolateral (a, b, c, d, e, e') chaetae; labial type AC, papillae A-E respectively with $1,4,0,3$, and 2 guard chaetae (Fig. 3D). Postlabial chaetae $4+4$ along ventral groove.

Body chaetotaxy. Ordinary chaetae differentiated in meso- and macrochaetae. Th. I tergum with $6+6$ chaetae dorsally (Fig. 3A). Th. II-III and Abd. I-III terga with three chaetae on both side of axial line and no dorsal axial chaetae. Abd. IV tergum with one dorsal axial chaeta $(\mathrm{m} 0)$, Abd. V without dorsal axial chaetae, Abd. VI with two axial chaetae (a0 and p0) (Fig. 3A). Th. I, II and III sterna without chaetae.

Appendages. Subcoxa 1 of legs I-III with 4, 5 and 5 chaetae, subcoxa 2 with 1, 4 and 4 chaetae respectively. Tibiotarsi of legs I, II and III with $20(1,8,11), 20(1,8$, 11 ) and $19(1,7,11)$ chaetae (Fig. 4B). Unguis without inner teeth, with lateral teeth. 

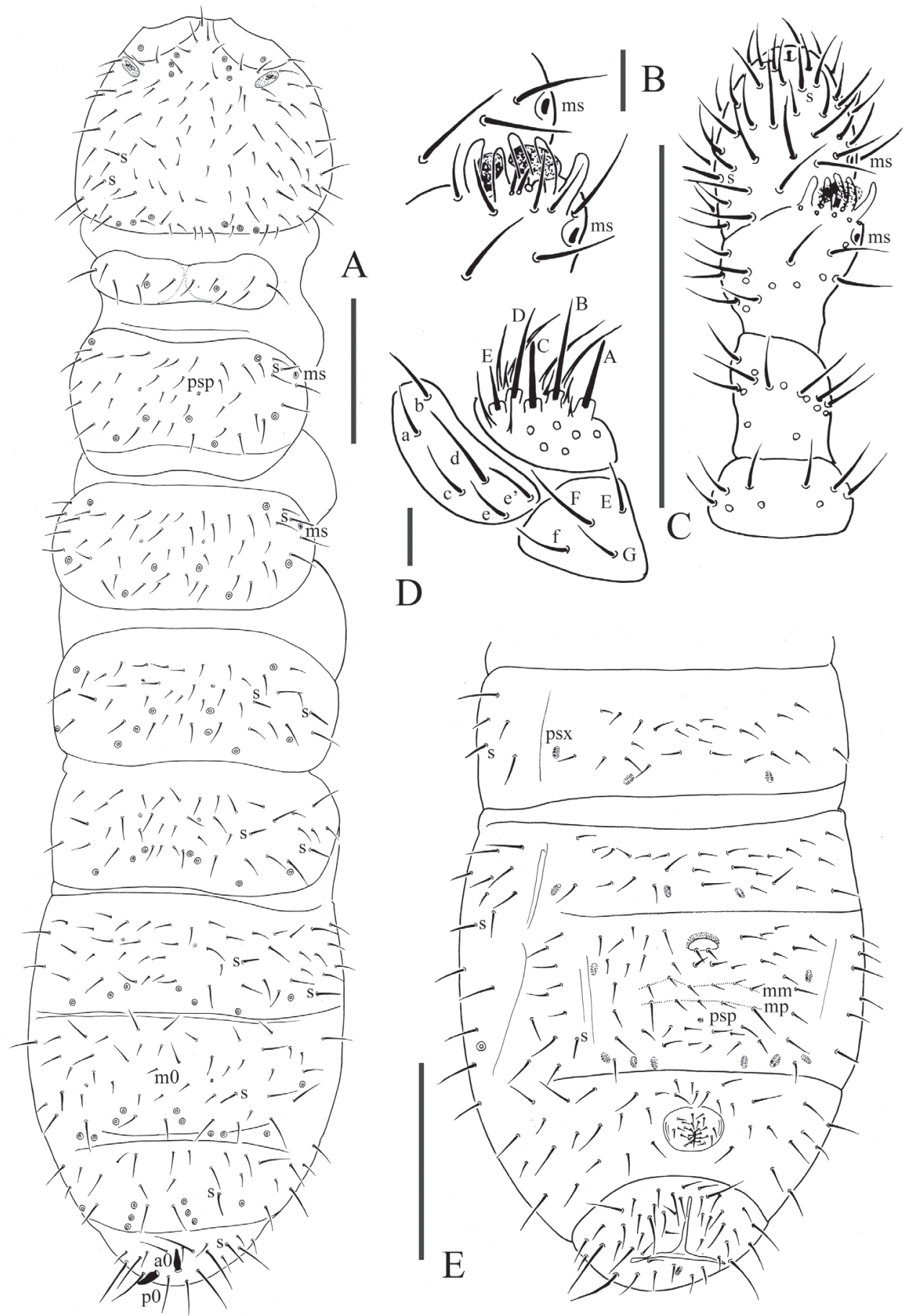

Figure 3. Oligaphorura pseudomontana sp. n. A dorsal side of body B organ of Ant. III C antenna D labium E ventral side of Abd. II-VI. Scales: $0.1 \mathrm{~mm}$ (A, C \& E), 0.01 (B \& D) 

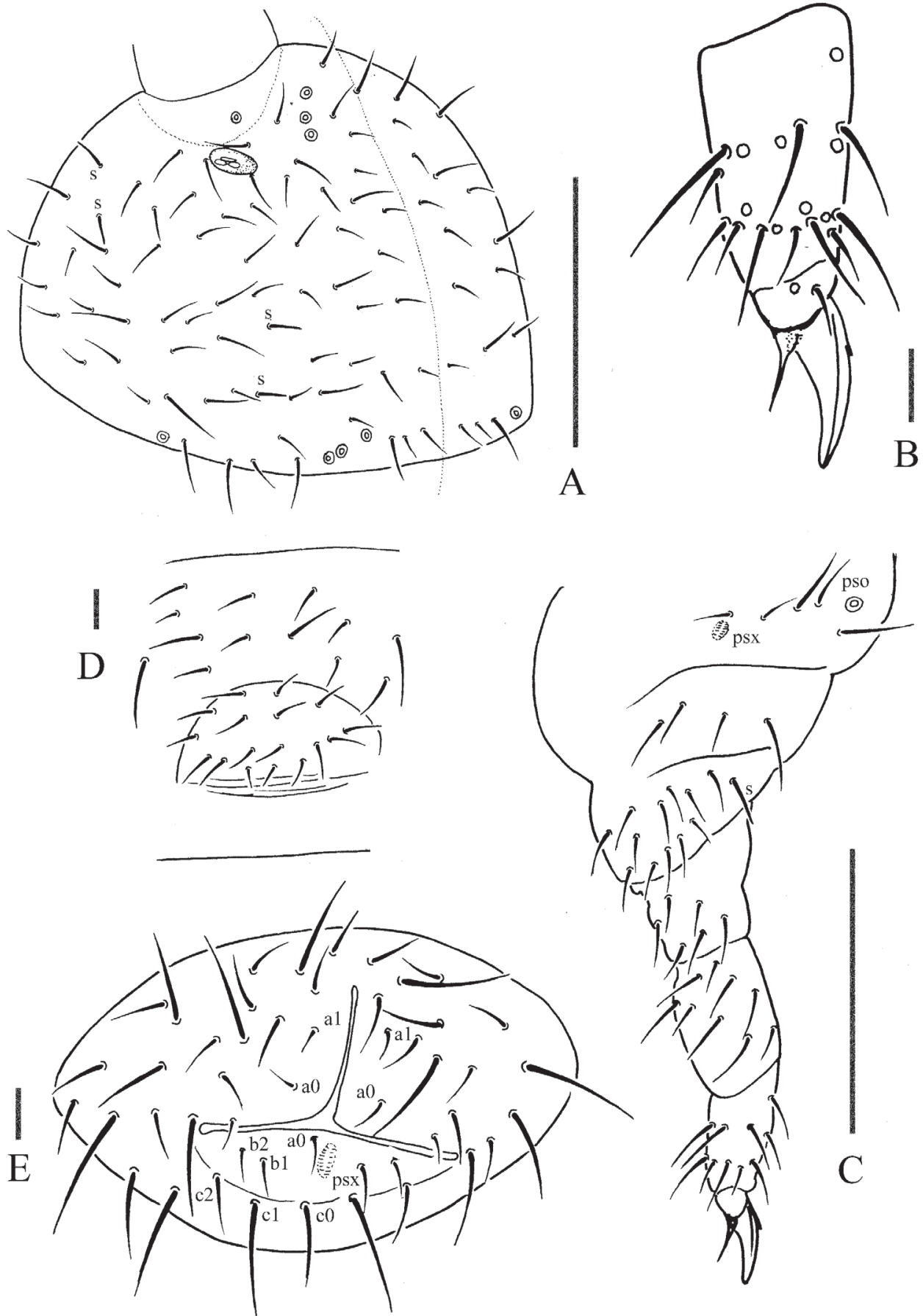

Figure 4. Oligaphorura pseudomontana sp. n. A dorsal side of head B distal part of leg III C leg III D female genital plate $\mathbf{E}$ anal valves. Scales: $0.1 \mathrm{~mm}(\mathrm{~A} \& \mathrm{C}), 0.01 \mathrm{~mm}(\mathrm{~B}, \mathrm{D} \& \mathrm{E})$ 
Unguiculus slender and pointed, 0.6 times as long as inner edge of unguis, with inner basal lamella (Fig. 4B). Ventral tube with 6-7+6-7 distal chaetae and $2+2$ basal chaetae, without anterior chaetae. Furca reduced to a small cuticular fold with $2+2$ dental chaetae in two rows posteriorly; two manubrial rows of chaetae present (Fig. 3E).

Female genital plate with 17 chaetae (Fig. 4D), male genital plate with 19-24 chaetae. Anal valves with numerous acuminate chaetae; each lateral valve with a 0 and 2a1; upper valves with chaetae a0, 2b1, 2b2, c0, 2c1, 2c2 (Fig. 4E). Anal spines present on indistinct papillae, 0.75 times as long as inner edge of unguis (Fig. 3A).

Etymology. Named for the similarity with the Korean species $O$. montana Weiner, 1994.

Ecology. Found in coniferous forest.

Remarks. The new species is very similar to the species montana collected in the mountain of North Korea, sharing the following characters: an increased number of pseudocelli dorsally; well marked base of antenna with 1 pseudocellus and 3 dorsal pseudocelli outside; subcoxa 1 of legs I-III with 1 pseudocellus each; dorsally S-chaetae formula as 11/011/22211 from head to Abd. V; S-microchaetae present on Th. II-III; head with chaeta p1 level with p2; Th. I, II and III sterna without chaetae; anal spines 0.75 times as long as inner edge of unguis; claw without inner teeth but with one lateral tooth; unguiculus with basal lamella. But they can be separated easily by the number of pseudocelli on Abd. V and VI terga (5 and 3 in montana versus 6 and 4 in pseudomontana sp. n.), parapseudocelli on the body (indistinct in montana, versus 00/000/222401 by half-sternite in pseudomontana sp. $\mathrm{n}$.), the number of chaetae on Th. I tergum $(7+7$ in montana versus $6+6$ in pseudomontana sp. n.), and the number of chaetae on tibiotarsi (19, 19, 18 in montana versus 20, 20, 19 in pseudomontana sp. n.).

\section{Acknowledgements}

Thanks should be given to Dr. Tang Xuguang (Northeast Institute of Geography and Agroecology, Chinese Academy of Sciences) who collected the specimens for our work. The present study was supported by the National Natural Sciences Foundation of China (NO. 31070467, 40901036, 41171047), Knowledge Innovation Programs of Chinese Academy of Sciences (KZCX2-YW-BR-16), and the Fundamental Research Funds for the Central Universities (NO. 2008-10008 and 2009-05022).

\section{References}

Bagnall RS (1949) Contribution toward a knowledge of the Onychiuridae (CollembolaOnychiuroidea). V-X. Annals and Magazine of Natural History 12: 498-511.

Bellinger PF, Christiansen KA, Janssens F (2012) Checklist of the Collembola of the World. Available from: http://www.collembola.org. 
D'Haese CA (2003) Homology and morphology in Poduromorpha (Hexapoda, Collembola). European Journal of Entomology 101: 385-407.

Fjellberg A (1984) Collmbola from Jan Mayen, Björnöya and Hopen with additions to the species list from Spitsbergen. Fauna norvegica Serie B 31: 69-76.

Fjellberg A (1987) Northern species of Onychiurus Gervais, 1841, subgenus Archaphorura Bagnall, 1949 (Collembola: Onychiuridae). Entomologica scandinavica 18: 279-288.

Fjellberg A (1999) The labial palp in Collembola. Zoologischer Anzeiger 237: 309-330.

Fjellberg A, Weiner MW (1994) Two interesting species of Onychiurinae (Collembola) from Ukraine and some remarks on Allaphorura franzi (Stach, 1946). Acta zoological cracoviensia 37: 59-64.

Massoud Z (1967) Monographie des Neanuridae, Collemboles Podurmorphes à pièces buccales modifiées. Biologie de l'Amérique Australe, CNRS, Paris, 7-399.

Sun X, Wu DH (2012) A Newly Recorded Tribe of family Onychiuridae (Collembola, Poduromorpha) with a new species and a newly recorded species from northeast China. Acta Zootaxonomica Sinica 37(1): 105-110.

Weiner MW (1994) Onychiurinae Bagnall of North Korea: species with small postantennal organs (Collembola, Onychiuridae). Bulletin de la Société entomologique de France 99(1): $31-40$.

Yoshii R (1996) Identity of some Japanese Collembola IV. "Deuteraphorura” Group of Onychiurus. Annals of the speleological research institute of Japan (Iwaizumi) 14: 1-15. 